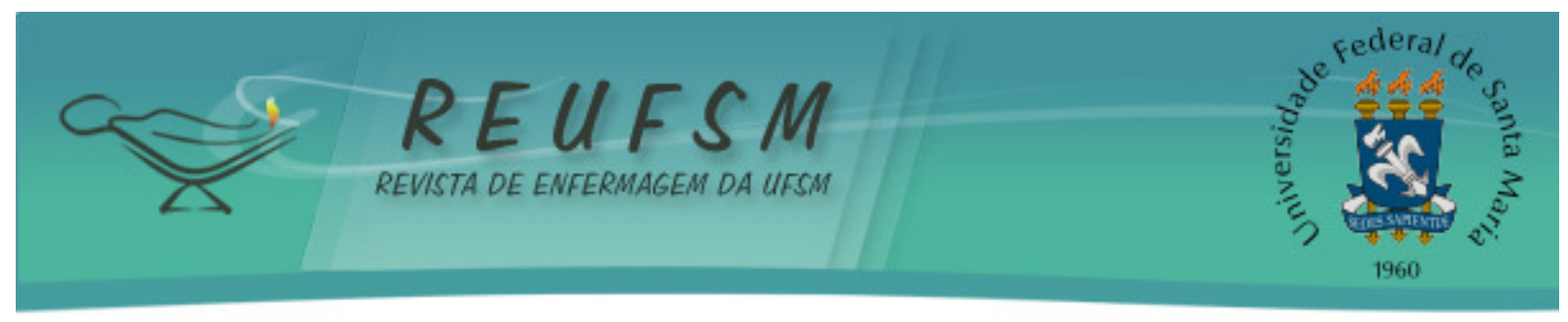

ARTIGO ORIGINAL

\title{
TECNOLOGIAS NA PROMOÇÃO DA SAÚDE DE IDOSOS COM DOENÇAS CRÔNICAS NA ATENÇÃO PRIMÁRIA À SAÚDE
}

\section{HEALTH PROMOTION TECHNOLOGIES IN ELDERLY PEOPLE WITH CHRONIC DISEASES IN PRIMARY HEALTH CARE}

\section{TECNOLOGÍAS DE PROMOCIÓN DE LA SALUD EN ANCIANOS CON ENFERMEDADES CRÓNICAS EN LA ATENCIÓN PRIMARIA DE SALUD}

\author{
Ana Alinne Gomes da Penha ${ }^{1}$ \\ Juliana Alexandra Parente Sa Barreto ${ }^{2}$ \\ Rosely Leyliane dos Santos ${ }^{3}$ \\ Regina Petrola Bastos Rocha ${ }^{4}$ \\ Huana Carolina Cândido Morais ${ }^{5}$ \\ Maria Corina Amaral Viana ${ }^{6}$
}

Doi: $10.5902 / 2179769217160$

RESUMO: Objetivo: conhecer as tecnologias empregadas pelos enfermeiros na promoção da saúde de idosos com doenças crônicas na Atenção Primária à Saúde. Método: pesquisa exploratória, com abordagem qualitativa e de caráter descritivo, realizada em uma cidade da região do Cariri, sul do Ceará, Brasil. Foram incluídos os enfermeiros da atenção primária que empregavam tecnologias para idosos com doença crônica. Utilizou-se entrevista semiestruturada para coleta de dados nos meses de julho e agosto de 2014, com análise de conteúdo temático. Resultados: emergiram duas categorias: Tecnologias empregadas na promoção da saúde de idosos com doenças crônicas e Estratégias para emprego de tecnologias no grupo de idosos na Atenção Primária à Saúde. Destacaram-se as tecnologias leves (acolhimento humanizado e vínculo) e duras (glicosímetro, tensiômetro e outras), que foram escolhidas pelos profissionais para as atividades de promoção da saúde. Conclusão: não existe clareza no conceito de tecnologias de saúde, especialmente no emprego das tecnologias leve-duras.

Descritores: Tecnologia; Promoção da saúde; Doenças crônicas; Idoso; Enfermagem.

ABSTRACT: Aim: to know the technology adopted by nurses in health promotion for elderly people with chronic diseases in Primary Health Care. Method: this is an exploratory research, with qualitative and descriptive approach, held at Cariri, south of Ceará, Brazil. Nurses who adopt technologies for the older adults with chronic were included. Semi-structured interview was used for data collection during July and August 2014, using thematic content analysis. Results: from the analysis, two categories emerged: Technologies used in health promotion of elderly people with chronic diseases

\footnotetext{
${ }^{1}$ Enfermeira. Especialista em Saúde da Pessoa Idosa. Membro do Grupo de Pesquisa Tecnologias em Saúde no SUS da Universidade Regional do Cariri. Crato, CE, Brasil. E-mail: alinne1304@hotmail.com

2 Enfermeira. Especialista em Assistência e Gestão em Saúde da Família. Membro do Grupo de Pesquisa Tecnologias em Saúde no SUS da Universidade Regional do Cariri. Crato, CE, Brasil. E-mail: julyannaparente@yahoo.com.br

${ }_{3}^{3}$ Enfermeira. Mestranda pelo Programa de Pós-Graduação em Enfermagem da Universidade Regional do Cariri. Crato, CE, Brasil. E-mail: leiliany.santos@bol.com.br

${ }^{4}$ Enfermeira. Mestre em Desenvolvimento Regional Sustentável. Docente do Curso de Enfermagem pela Universidade Regional do Cariri. Crato, CE, Brasil. E-mail: rpetrola@yahoo.com.br

${ }^{5}$ Enfermeira. Doutoranda pelo Programa de Pós-Graduação em Enfermagem da Universidade Federal do Ceará. Fortaleza, CE, Brasil. E-mail: huanacarolina88@gmail.com

${ }^{6}$ Enfermeira. Doutora em Enfermagem. Líder do Grupo de Pesquisa Tecnologias em Saúde no SUS da Universidade Regional do Cariri. Crato, CE, Brasil. E-mail: corina.viana@urca.br
} 


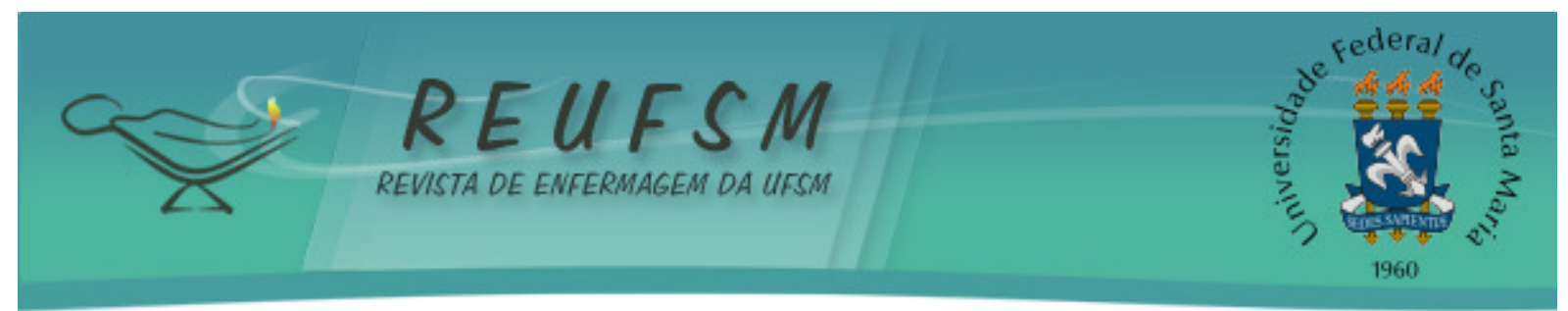

and Strategies for the use of technologies in the elderly group in Primary Health Care. Light technologies stood out (humanized host and bond) and hard (glucose meter, tensiometer among others). They were chosen for health promotion activities by professionals. Conclusion: there is no clarity on the concept of health technologies, especially in the use of soft-hard technologies.

Descriptors: Technology; Health promotion; Chronic disease; Aged; Nursing.

RESUMEN: Objetivo: conocer la tecnología empleada por las enfermeras en la promoción de la salud para ancianos con enfermedades crónicas en la Atención Primaria de Salud. Método: investigación exploratoria, cualitativa y descriptiva, realizada en Cariri, ciudad del sur de Ceará, Brasil. Se incluyó enfermeras de atención primaria que emplean tecnologías para ancianos con enfermedades crónicas. Fue utilizada para la recolección de datos una entrevista semi-estructurada realizada entre julio y agosto 2014. Resultados: a partir del análisis de las categorías surgieron: tecnologías empleadas en la salud para ancianos con enfermedades crónicas y estrategias para el empleo de tecnologías para ancianos en la Atención Primaria de Salud. Se destacaron las tecnologías leves (anfitrión humanizado y bonos) y severas (medidor de glucosa, tensiómetro y otros) que fueron elegidas para actividades de promoción de la salud por los profesionales. Conclusión: no hay claridad sobre el concepto de tecnologías de la salud, especialmente en el uso de blandas-duras.

Descriptores: Tecnología; Promoción de la salud; Enfermedad crónica; Anciano; Enfermería.

\section{INTRODUÇÃO}

A população mundial está envelhecendo de forma gradual, com o aumento da expectativa de vida, elevando os riscos de adoecimento por Doenças Crônicas Não Transmissíveis (DCNT). Em 2010, o Brasil apresentava uma população de 190.755.799 habitantes, dos quais 20.590.599 eram considerados idosos (idade $\geq 60$ anos), correspondendo a $10,8 \%$ da população brasileira. ${ }^{1}$ Esses dados apontam que o país caminha para um perfil demográfico mais envelhecido, caracterizado por uma transição epidemiológica e demográfica, em que as DCNT ocupam lugar de destaque, em detrimento das doenças infecciosas e contagiosas. ${ }^{2}$

Nesse contexto, a promoção da saúde consiste em desenvolver mudanças e práticas saudáveis para o indivíduo e deve ser incorporada na assistência contínua dos profissionais da saúde, evitando possíveis agravos. A promoção da saúde pode ser definida como o conjunto de medidas destinadas a ter uma boa saúde, promover a qualidade de vida, reduzir vulnerabilidades e riscos relacionados aos seus determinantes e condicionantes modos de viver, condições de trabalho, habitação, ambiente, educação, lazer, cultura, acesso a bens e serviços essenciais. ${ }^{3}$

As medidas preventivas que estão na proposta de promoção da saúde distinguem-se em quatro níveis: primário, secundário, terciário e quaternário. Nos quatro níveis, podem ser utilizados quatro tipos diferentes de abordagens tecnológicas para efetuar intervenções preventivas, a saber: aconselhamento ou mudança de estilo de vida, rastreamento, quimioprevenção (uso de drogas que, comprovadamente, reduzem o risco de doenças e/ou suas complicações) e imunização. ${ }^{4}$

Entende-se como tecnologia o resultado de processos firmados, que devem ser verificados e controlados sistematicamente, pelas experiências do cotidiano e da pesquisa, pelo desenvolvimento de um conjunto de conhecimentos científicos para a construção de produtos materiais, ou não, com o objetivo de provocar intervenções sobre uma situação prática. ${ }^{5}$ 


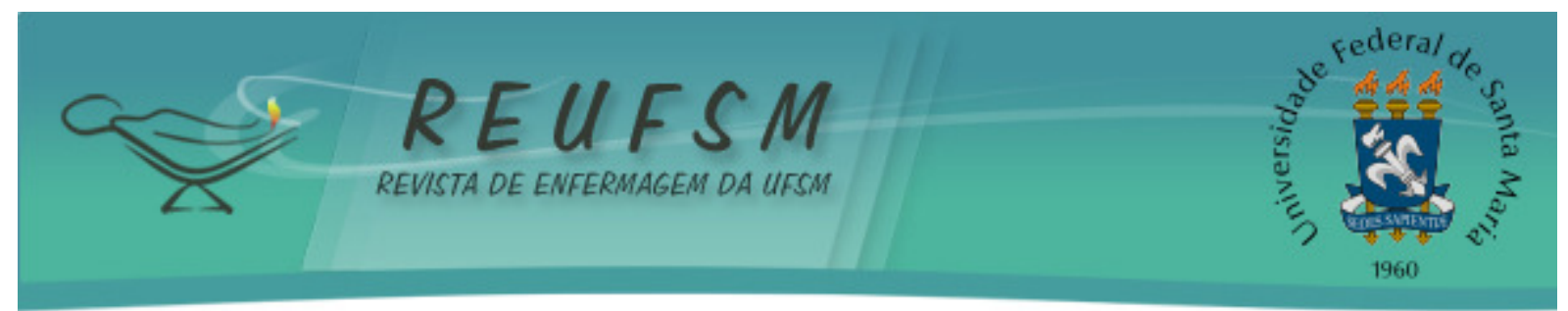

Ainda, segundo a Portaria 2.510/GM de 19 de dezembro de 2005, podem ser consideradas tecnologias em saúde os sistemas organizacionais, educacionais, de informações e de suporte, programas e protocolos assistenciais, por meio dos quais a atenção e os cuidados com a saúde são prestados à população. ${ }^{6}$

A tecnologia faz-se presente no processo de cuidar de enfermagem em todos os aspectos. Ademais, a mesma se apresenta como se estabelecem as relações entre os atores e na forma como se dá o cuidado em saúde, sendo este compreendido como um trabalho vivo em ato. ${ }^{7}$

Existem diferentes métodos para se caracterizar as tecnologias, entretanto a classificação mais difundida é aquela que considera tecnologias leves, nas quais se visualiza claramente que a implementação do cuidado requer o estabelecimento de relações (vínculo, gestão de serviços e acolhimento); tecnologia leve-dura, quando lança mão de saberes estruturados (teorias, modelos de cuidado, processo de enfermagem); e tecnologia dura, que utiliza instrumentos, normas e equipamentos tecnológicos. ${ }^{8}$ Essa classificação aborda a tecnologia de forma abrangente, desde o processo de vínculo entre profissional e cliente até o produto final do cuidado, por isso foi adotada como referencial para este estudo.

O enfermeiro tem papel fundamental no acompanhamento de idosos com doenças crônicas seja na promoção, proteção e recuperação da saúde. Em virtude disso, faz-se o seguinte questionamento: quais as tecnologias empregadas pelos enfermeiros na promoção da saúde de idosos com doenças crônicas na Atenção Primária à Saúde (APS)?

0 assunto em questão foi escolhido devido à importância do desenvolvimento de estratégias de promoção da saúde para aumentar o nível de conhecimento das pessoas, a fim de que elas busquem solucionar problemas e complicações futuras. Essa situação é ainda mais presente nos idosos que, por condições fisiológicas e sociais, são mais suscetíveis ao adoecimento.

Ademais, a temática torna-se importante, pois delimitar as tecnologias empregadas por profissionais de saúde que atendem idosos com doenças crônicas pode ser um indicativo de quais estratégias são mais efetivas para o cuidado prestado a esse indivíduo. Ainda, fornece aos gestores indicativos da necessidade de capacitar os profissionais nessa área. Salienta-se que poucos estudos que abordam as tecnologias foram identificados na literatura, apesar de sua importância para a estruturação da atenção primária ao idoso diagnosticado com doenças crônicas.

Considerando os aspectos mencionados, o presente estudo teve como objetivo conhecer as tecnologias empregadas pelos enfermeiros na promoção da saúde de idosos com doenças crônicas na APS.

\section{MÉTODO}

O estudo representa uma pesquisa exploratória com abordagem qualitativa de caráter descritivo. Foi realizado com enfermeiros da APS de um município de pequeno porte da região do Cariri, sul do Ceará, Brasil.

Os participantes da pesquisa foram nove enfermeiros que atuam nas Unidades Básicas de Saúde (UBS) do referido município. O cenário do estudo constituiu-se por duas UBS da zona urbana e sete UBS da zona rural.

Os critérios de inclusão para participação na pesquisa foram os enfermeiros que empregam tecnologias de promoção à saúde no cuidado a idosos portadores de doenças crônicas tratáveis na APS. A presença desse critério foi determinado pela pesquisadora durante a entrevista, quando identificou que todos os enfermeiros empregavam algum tipo de tecnologia no cuidado ao idoso. Assim, todos estavam aptos e participaram da pesquisa, 


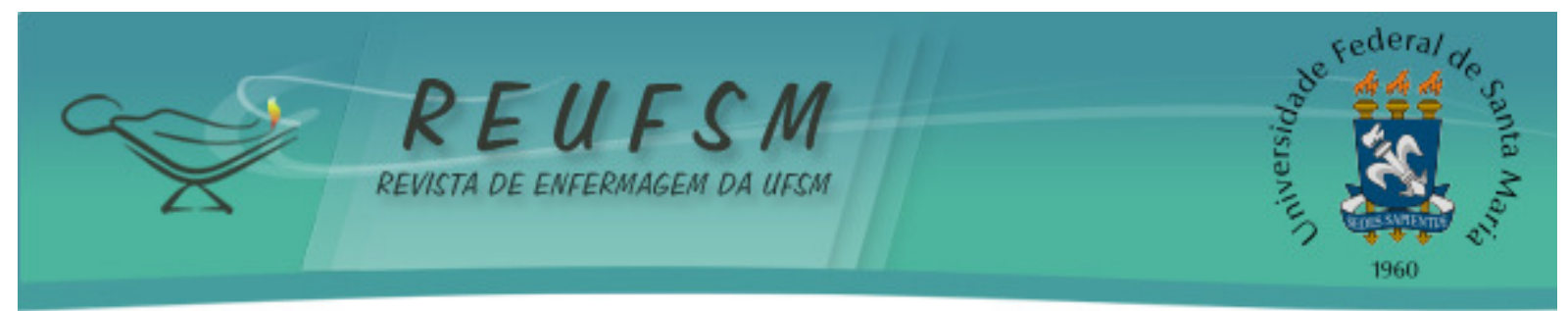

portanto não foi necessário o estabelecimento de critérios de exclusão. A pesquisa foi desenvolvida durante os meses de julho e agosto de 2014.

0 instrumento de coleta de dados adotado foi uma entrevista semiestruturada na qual o entrevistador tem liberdade para direcionar a situação como considerar adequada, podendo explorar mais amplamente a questão. ${ }^{9}$ Os entrevistados foram os enfermeiros da APS. As entrevistas foram agendadas e realizadas nos locais de trabalho dos mesmos. Foi utilizado gravador digital e, posteriormente, feita a transcrição e a digitação das falas.

Antes da entrevista, foi realizada a leitura e a assinatura da autorização individual do Termo de Consentimento Livre e Esclarecido (TCLE). É importante esclarecer que para zelar pela identidade dos participantes e pelo sigilo absoluto das informações prestadas, foram utilizados códigos como a letra $\mathrm{E}$ (Enfermeiro) e números de identificação 1, 2... 9 . Duas questões nortearam a entrevista, o conhecimento acerca das tecnologias e como as mesmas eram empregadas na atuação profissional dos entrevistados.

Utilizou-se a técnica de análise de conteúdo temático a qual é dividida em três etapas: a pré-análise, que corresponde à escolha do material a ser utilizado; exploração do material, que consiste na operação de codificação; tratamento dos resultados obtidos e interpretação, que coloca em relevo as informações obtidas, propõe inferências e realiza interpretações previstas no seu quadro teórico. ${ }^{10}$

Durante a análise de conteúdo temático dos dados emergiram duas categorias, a saber: tecnologias empregadas na promoção da saúde de idosos com doenças crônicas e estratégias para emprego de tecnologias no grupo de idosos na APS.

O projeto foi encaminhado ao Comitê de Ética em Pesquisa (CEP) da Universidade Federal do Ceará, obedecendo à resolução $n^{\circ}$ 466/12 do Conselho Nacional de Saúde (CNS), que rege as pesquisas envolvendo seres humanos ${ }^{11}$, obtendo parecer favorável (protocolo $\left.n^{\circ} 660.902 / 2014\right)$.

\section{RESULTADOS E DISCUSSÃO}

No perfil dos entrevistados, a faixa etária situou-se entre 24 e 34 anos, configurando-se adultos jovens em atuação. Em relação ao sexo, foi possível observar que a maioria era composta pelo sexo feminino. 0 tempo de trabalho foi superior a dois anos de serviço pela maioria dos enfermeiros. No tocante à titulação, a maioria dos participantes respondeu que possuía pós-graduação. Vale destacar que a área de escolha foi variada, sendo que apenas um entrevistado possuía em saúde da família.

A seguir, são apresentados os trechos das entrevistas que contemplam as duas categorias e a discussão com a literatura pertinente.

\section{Tecnologias empregadas na promoção da saúde de idosos com doenças crônicas}

Considerando a classificação de tecnologias para a promoção da saúde adotadas para o estudo, a saber, leve, leve-dura e dura $^{8}$, quando os enfermeiros foram indagados quanto às empregadas na promoção da saúde do idoso com doença crônica foram citados os três tipos acima mencionados. A seguir são apresentados os discursos dos enfermeiros que utilizam as leves:

nosso trabalho só é possível de desenvolver se tiver uma relação de confiança e respeito, principalmente, com os pacientes idosos na hora das orientações para mudanças de hábitos na alimentação. (E1) 


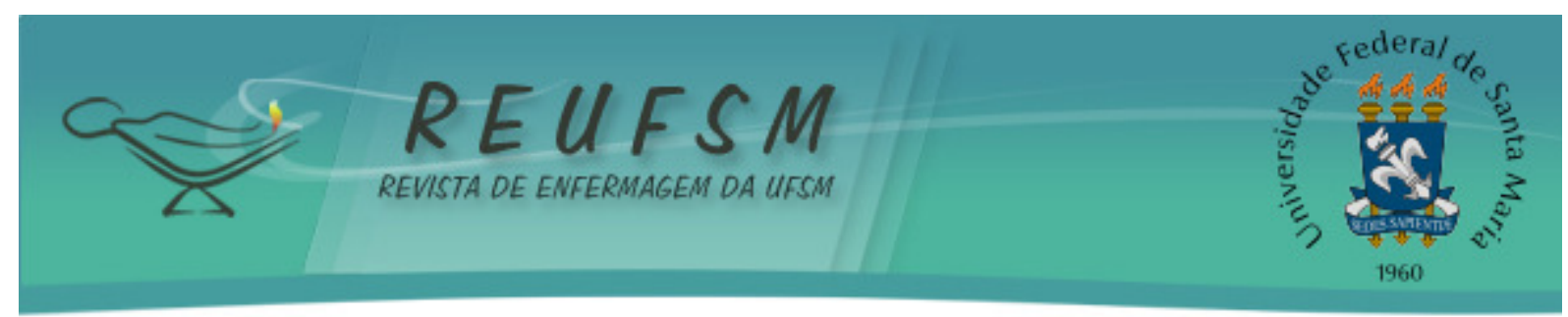

o contato diário na unidade de saúde permite criar um vínculo com os idosos e desenvolver um ambiente mais favorável com acolhimento humanizado. (E3)

Sabe-se que as tecnologias leves condensam em si as relações de interação e subjetividade, o que dinamiza o processo do cliente no grupo, de modo a facilitar a aprendizagem. Ao se privilegiar o diálogo, a escuta e o envolvimento com a queixa do outro, tem-se um acolhimento resolutivo, sendo imperiosa a corresponsabilização entre profissionais de saúde e cliente na procura pelo melhor cuidado. ${ }^{12}$

Pode estar associada ao uso das tecnologias leves a mudança de hábitos de vida, que geram redução nas taxas de mortalidade por doenças cardiovasculares e respiratórias crônicas. Essa diminuição está associada possivelmente ao resultado do controle do tabagismo e do maior acesso à APS. As DCNT são um problema de saúde global e uma ameaça à saúde e ao desenvolvimento humano. Às doenças cardiovasculares, respiratórias crônicas, renais, diabetes e cânceres são atribuídos cerca de $70 \%$ dos óbitos em $2007 .{ }^{13}$

A APS está organizada a partir das equipes da estratégia saúde da família (ESF) e traz como proposta a multidisciplinaridade para atender a comunidade adstrita em seu território. Tal estratégia tem como um dos objetivos a promoção da saúde e prevenção de doenças. As intervenções sobre a população que não apresentam doenças, mas com fatores de risco para adoecer, precisam ser direcionadas como suporte e apoio às mudanças de estilo de vida e na incorporação de um modelo de vida saudável. ${ }^{14}$

Entretanto, para que as equipes possam incorporar novas tecnologias nos processos de trabalho, faz-se necessário superar o modelo assistencial existente nos serviços de saúde, de forma que os doentes possam ter acesso aos recursos disponíveis na rede pública. Parte-se da premissa que o vínculo se constitui elemento fundamental e estruturante das práticas de saúde. ${ }^{15}$

Apesar da importância das tecnologias leves para o controle das doenças crônicas em idosos, alguns profissionais não as citaram como relevantes, pois consideraram as tecnologias que utilizam recursos materiais e palpáveis como mais empregadas. Assim, os discursos dos entrevistados revelaram uma limitação na classificação de tecnologias leveduras quando citaram apenas os instrumentos nas atividades de educação em saúde com idosos. Logo, não conceituaram tecnologias leve-duras como a construção de um saber estruturado.

[...] palestras educativas, a caderneta do idoso, trabalhos em grupos com produção de cartazes e panfletos e pinturas que têm participação dos profissionais do CRAS, prevenção de quedas $e$ fraturas, alimentação saudável e atividade física. (E3)

bom, no trabalho feito com os idosos na promoção da saúde nós utilizamos basicamente palestras, dinâmicas, rodas de conversa, material audiovisual, pôsteres, ilustrações, panfletos informativos e explicativos. (E1)

Um aspecto a ser considerado na utilização dos instrumentos citados nas falas dos entrevistados é a sua correta adequação, conforme as limitações físicas, psicológicas e sociais dos clientes, alcançando, assim, sua finalidade. As tecnologias leve-duras permitem processar o olhar do profissional sobre o usuário, como objeto de sua intervenção, em um processo de apreensão de seu mundo e de suas necessidades a partir de ponto de vista construído por saberes bem definidos. ${ }^{8}$ 


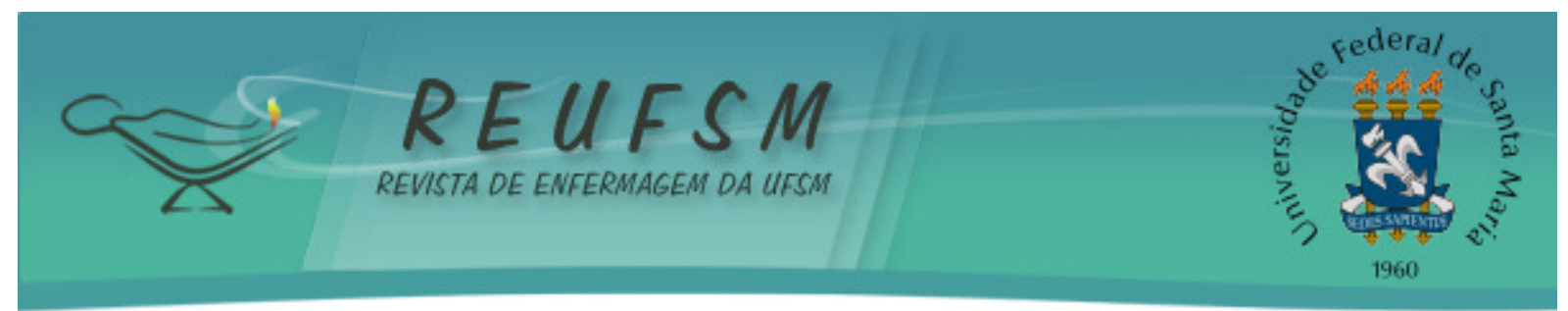

Foi possível observar que os entrevistados citaram a participação dos profissionais do Núcleo de Apoio à Saúde da Família (NASF) e do Centro de Referência de Assistência Social (CRAS). Dessa forma, a promoção da atividade física e da nutrição saudável possibilita rever crenças e valores em seus contextos de vida, que cada vez mais são limitados pela influência dos fatores econômicos, políticos, sociais, culturais e a visão de mundo das pessoas. ${ }^{16}$

O Sistema Único de Saúde (SUS), conforme ratificado na Política Nacional de Promoção da Saúde, deve estimular e desencadear ações que favoreçam o compromisso da sociedade e de seus dirigentes com a ampliação da autonomia de sujeitos e coletividades, fortalecendo sua capacidade crítica de análise do seu contexto sociocultural e econômico e, consequentemente, propiciando e facilitando o acesso às escolhas mais saudáveis. ${ }^{17}$

As tecnologias duras também são utilizadas como instrumentos de trabalho dos profissionais, assim como as tecnologias para a prevenção de agravos, a exemplo das campanhas de vacinação e dos exames de rastreamento para monitorar, diagnosticar e tratar os idosos com enfermidades crônicas, principalmente, hipertensos e diabéticos que representam a maioria dessa clientela e possuem um maior contato com os profissionais da APS.

[...] também dentro do propósito de promover saúde, foco na prevenção e rastreamento com utilização de verificação de glicemia capilar com glicosímetro. Levo, também, o tensiômetro para aferição da pressão arterial, a balança para peso, calculadora para fazer o IMC, fita métrica para medição da cintura, campanhas em praças públicas a respeito da fragilidade do idoso acometido pelas doenças crônicas com ênfase em diagnóstico precoce e também alertar para os perigos dessas comorbidades na velhice. (E4)

[...] realizo campanhas para prevenção de complicações nos pacientes hipertensos e diabéticos com utilização de aparelho de pressão e glicosímetro para medir a glicemia. (E6)

Os instrumentos de rastreio apresentam algumas vantagens: normalmente, são de fácil e rápida aplicação, de baixo custo, podem ser utilizados por profissionais de saúde não especialistas, desde que esses sejam treinados e, ainda, apresentam boa aceitação tanto pela população avaliada quanto pelos avaliadores. Assim, esses instrumentos podem ser adotados pela equipe de saúde na APS como ferramentas para identificação das grandes síndromes geriátricas, facilitando a identificação dos casos e possibilitando a intervenção precoce, 0 acompanhamento e 0 direcionamento racional do fluxo assistencial. $^{18}$

Dessa forma, a literatura científica na área e as experiências de outros países destacam que as tecnologias mais efetivas para a redução da carga de DCNT são aquelas que trabalham com os fatores determinantes e condicionantes da saúde individual e coletiva, elaborando políticas públicas orientadas pela ótica da integralidade e adequadas às realidades e necessidades de cada região de saúde. ${ }^{19}$

Logo, percebe-se que os enfermeiros investigados afirmaram conhecer e utilizar os diferentes tipos de tecnologias disponíveis para o cuidado dos idosos com doenças crônicas. Entretanto, percebeu-se pelos relatos que essas estratégias não são direcionadas para o cuidado individual de cada idoso. Ademais, não foi possível identificar a periodicidade das ações, apesar de acreditar-se que as mesmas não são realizadas de forma contínua. 


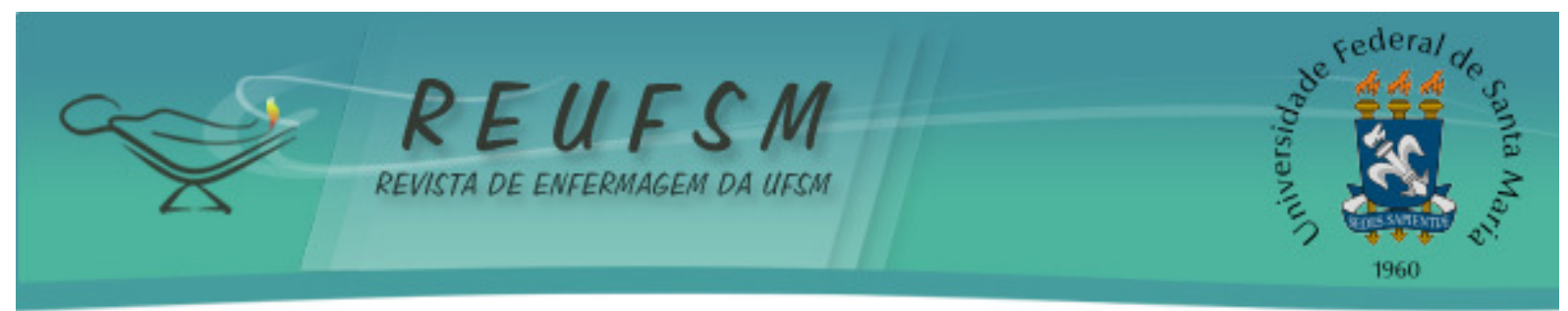

Estratégias para emprego de tecnologias no grupo de idosos na atenção primária à saúde

Os quatro grupos de DCNT de maior impacto mundial são doenças cardiovasculares, diabetes mellitus, neoplasias e doenças respiratórias crônicas. Tais grupos apresentam quatro fatores de risco em comum: tabagismo, inatividade física, alimentação não saudável e abuso de álcool. ${ }^{20} \mathrm{~A}$ seguir, são apresentadas estratégias utilizadas pelos enfermeiros para promoção da saúde desses grupos predisponentes:

[...] as visitas domiciliares, busca ativa, os agentes comunitários de saúde ajudam na divulgação na comunidade das reuniões do grupo de idosos hipertensos e diabéticos. (E3)

as estratégias são diversas, a abordagem direta às famílias e ao idoso como realização de encontros dos grupos de idosos e toda equipe da estratégia saúde da família. A equipe do NASF dá bastante suporte e os profissionais do CRAS também colaboram nas atividades dos pacientes idosos. (E2)

A atuação das equipes de saúde da família é essencial para o fortalecimento das ações de promoção da saúde no cuidado integral das DCNT, pois o desenvolvimento de suas atividades tem como fundamentos a efetivação da integralidade, o estímulo à participação popular e ao controle social, as relações de vínculo e responsabilização e, principalmente, a promoção da equidade. ${ }^{19}$

A partir dos discursos listados, observou-se que as estratégias são consideradas importantes para os enfermeiros e para os membros da equipe de saúde. Entretanto, não foram apresentadas ações efetivas ou resultados concretos de sua execução. Os grupos compostos por profissionais e idosos com hipertensão e diabetes foi o único exemplo. Acredita-se que ações direcionadas para a educação em saúde, abordando não só os assuntos mais recorrentes, hipertensão arterial e diabetes mellitus, como os já citados em atividades educativas e com uma periodicidade maior, abrangendo todos os segmentos da população, alcançassem resultados mais eficazes e efetivos para a promoção da saúde desses idosos.

Reorientar os modelos de atenção à saúde requer a compreensão da situação de saúde de determinado local para poder atuar na realidade de saúde. Em se tratando das DCNT, elas têm suas especificidades, especialmente no que diz respeito à qualidade de vida das pessoas e de suas famílias. Portanto, nessa perspectiva, há de se destacar o controle integrado e a tomada de decisão baseada em evidências científicas. ${ }^{19}$

0 trabalho dos profissionais de saúde da APS, sendo o enfermeiro um agente fundamental na construção da saúde, é fundamental para o desenvolvimento dessas tecnologias. ${ }^{21}$ Sendo assim, é importante para a APS o uso das tecnologias na prática cotidiana do trabalho.

\section{CONCLUSÃO}

Diante dos resultados obtidos pelo presente estudo, foi possível conhecer as tecnologias empregadas pelos enfermeiros na promoção da saúde dos idosos com doenças crônicas na APS. Dentre as tecnologias, vale destacar as tecnologias leves (acolhimento humanizado e vínculo) e duras (glicosímetro, tensiômetro, balança e fita métrica) que foram escolhidas para as atividades de promoção da saúde pelos profissionais. Em relação 


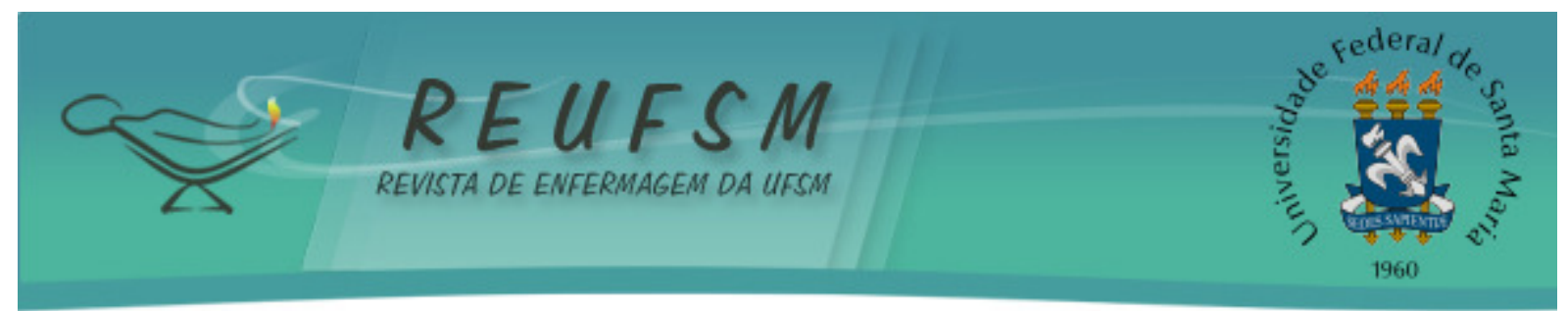

às tecnologias leve-duras, os entrevistados direcionaram suas respostas para os instrumentos como: palestras, dinâmicas, rodas de conversa, material audiovisual, pôsteres, ilustrações, panfletos informativos e explicativos, apresentando um conceito limitado sobre as mesmas.

Além disso, os entrevistados relataram empregar os três tipos de tecnologias segundo o conceito de Merhy. Esse fato ressalta que todas as tecnologias são igualmente importantes e devem estar inseridas nas diversas formas de trabalho da enfermagem bem como na promoção da saúde.

Ainda, o estudo limitou-se pela falta de conhecimento sobre a definição de tecnologias na saúde por parte dos entrevistados, que pode ter influenciado a interpretação e a análise dos dados. Ressalta-se que, devido à flexibilidade da entrevista semiestruturada, as definições foram fornecidas pelas pesquisadoras ao longo da entrevista.

Assim, a pesquisa aponta a necessidade de novos estudos que aprofundem os aspectos referentes às tecnologias em saúde. Para o ensino, o estudo pode contribuir com a formação de profissionais de enfermagem mediante inserção da temática na grade curricular tanto no nível de graduação como pós-graduação. E para a prática, poderá servir de guia para o emprego dessas tecnologias no cotidiano dos enfermeiros e da sociedade ou dos usuários do SUS.

\section{REFERÊNCIAS}

1. Instituto Brasileiro de Geografia e Estatística (IBGE). Censo 2010 [acesso em 2014 abr 10]. Disponível em: www.ibge.gov.br.

2. Moraes EN. Atenção à saúde do idoso: aspectos conceituais. Brasília: Organização PanAmericana da Saúde; 2012.

3. Heflin MT, Schmader KE, Sokol HN. Geriatriche health maintenance. UpToDate [Internet]. 2011 [acesso em 2014 maio 4]. Disponível em: www.uptodate.com/contents/geriatric-health-maintenance.

4. Russel $\mathrm{H}$, Fletcher RH, Sokol HN. Overview of preventive medicine in adults. UpTo Date [Internet]. 2011 [acesso em 2014 jul 25]. Disponível em: http: // www.uptodate.com.

5. Nietsche EA. As tecnologias assistenciais, educacionais e gerenciais produzidas pelos docentes dos cursos de enfermagem das instituições de ensino superior de Santa Maria-RS. Relatório Final. Santa Maria (RS): Universidade Federal de Santa Maria-UFSM/CNPq; 2003.

6. Brasil. Ministério da Saúde. Portaria 2.510, de 19 de dezembro de 2005. Institui Comissão para Elaboração da Política de Gestão Tecnológica no âmbito do Sistema Único de Saúde - CPGT. Diário Oficial da União, Brasília; 2005 dez 20. Seção 1, p. 77.

7. Rocha PK, Prado ML, Wal ML, Carraro TE. Cuidado e tecnologia: aproximações através do Modelo do Cuidado. Rev Bras Enferm. 2008;61(1):113-6.

8. Merhy, EE. Em busca de ferramentas analisadoras das tecnologias em saúde: a informação e o dia a dia de um serviço, interrogando e gerindo trabalho em saúde. In: Merhy EE, Onoko R, organizadores. Agir em saúde: um desafio para o público. $2^{\mathrm{a}}$ ed. São Paulo: Hucitec; 2002. p. 113-50.

9. Marconi MA, Lakatos EM. Fundamentos de metodologia científica. $7^{a}$ ed. São Paulo: Atlas; 2010. 


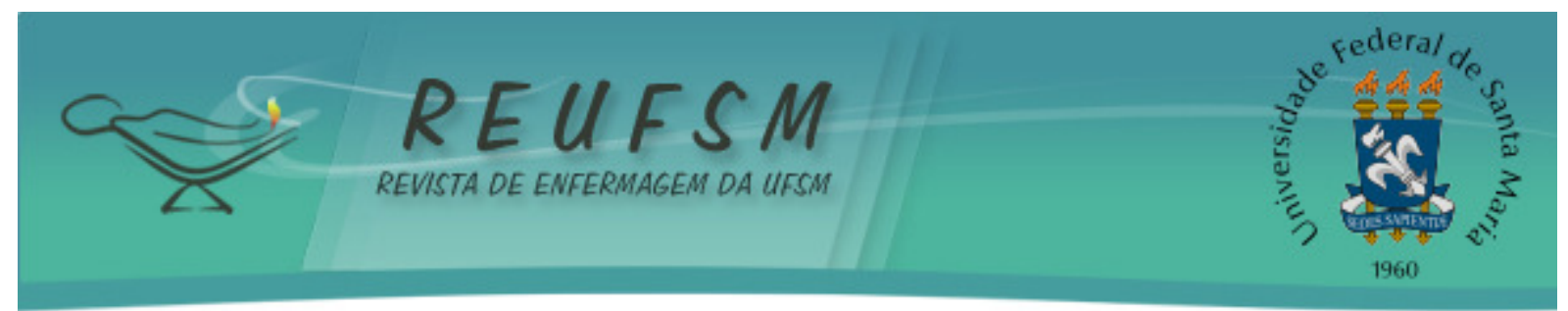

10. Minayo MCS. O desafio do conhecimento: pesquisa qualitativa em saúde. $11^{\text {a }}$ ed. São Paulo: Hucitec; 2010.

11. Brasil. Ministério da Saúde. Conselho Nacional de Saúde. Resolução $n^{\circ}$ 466, de 12 de dezembro de 2012. Diretrizes e normas regulamentadoras de pesquisa envolvendo seres humanos [Internet]. Brasília; 2012 [acesso em 2014 jul 10]. Disponível em: http: / / conselho.saude.gov.br/resolucoes/2012/Reso466.pdf.

12. Santos AM, Assis MMA, Rodrigues AAAO, Nascimento MAA, Jorge MSB. Linhas de tensões no processo de acolhimento das equipes de saúde bucal do Programa Saúde da Família: o caso de Alagoinhas, Bahia, Brasil. Cad Saúde Pública. 2007;23(1):75-85.

13. Schmidt MI, Duncan BB, Silva GA, Menezes AM, Monteiro CA, Barreto SM, et al. Chronic non communicable diseases in Brazil: burden and current challenges. Lancet. 2011;377(9781):1949-61.

14. Rose G. Estratégias da medicina preventiva. Porto Alegre: Artmed; 2010.

15. Brunello MEF, Cerqueira DF, Pinto IC, Arcênio RA, Gonzales RIC, Villa TCS, et al. Vínculo doente-profissional de saúde na atenção a pacientes com tuberculose. Acta Paul Enferm. 2009;22(2):176-82.

16. Fernandes MTO, Silva LB, Soares SM. Utilização de tecnologias no trabalho com grupos de diabéticos e hipertensos na Saúde da Família. Cienc Saude Colet. 2011;16 Supl 1:133140.

17. Brasil. Ministério da Saúde. Secretaria de Vigilância em Saúde. Secretaria de Atenção à Saúde. Política Nacional de Promoção da Saúde. $3^{a}$ ed. Brasília: Ministério da Saúde; 2010.60 p. (Série B. Textos Básicos de Saúde) (Série Pactos pela Saúde 2006; v. 7).

18. Cruz DT. A importância dos instrumentos de rastreio para a população idosa no contexto da APS. Editorial. Rev APS. 2014;17(1):5.

19. Brasil. Ministério da Saúde. Secretaria de Vigilância à Saúde. Secretaria de Atenção à Saúde. Diretrizes e recomendações para o cuidado integral de doenças crônicas não transmissíveis: promoção da saúde, vigilância, prevenção e assistência. Brasília: Ministério da Saúde; 2008. 72 p. (Série B. Textos Básicos de Atenção à Saúde) (Série Pactos pela Saúde 2006; v. 8).

20. Brasil. Ministério da Saúde. Secretaria de Atenção à Saúde. Departamento de Atenção Básica. Estratégias para o cuidado da pessoa com doença crônica. Brasília: Ministério da Saúde; 2014. 162 p. (Cadernos de Atenção Básica, n. 35).

21. Wild CF, Silveira A, Rosa EO, Favero NB, Gueterres EC, Leal SDS. Educação em saúde na sala de espera de uma policlínica infantil: relato de experiência. Rev Enferm UFSM [Internet]. 2014 [acesso em 2015 jan 10];4(3):660-6. Disponível em: http://cascavel.ufsm.br/revistas/ojs-2.2.2/index.php/reufsm/article/view/12397/pdf.

Data de recebimento: 07/03/2015

Data de aceite: 23/06/2015

Contato do autor responsável: Ana Alinne Gomes da Penha

Endereço postal: Rua Coronel Antônio Luiz, 1161 - Pimenta. CEP: 63105-000. Crato, CE, Brasil.

E-mail: alinne1304@hotmail.com 